\title{
Deterministic cooperative hybrid ring-mesh network coding for big data transmission over lossy channels in $5 \mathrm{G}$ networks
}

\author{
Hani H. Attar ${ }^{1}$, Ahmad A. A. Solyman², Ayat Alrosan ${ }^{3}$, Chinmay Chakraborty ${ }^{4}$ and Mohammad R. Khosravi $i^{*}$
}

\section{*Correspondence:}

m.r.khosravi.taut@gmail.com

${ }^{5}$ Department of Computer

Engineering, Persian Gulf

University, Bushehr, Iran

Full list of author information

is available at the end of the

article

\begin{abstract}
Wired and wireless communication data is getting bigger and bigger at such a high pace. Accordingly, the big data (BD) communication networks should be developed as quickly as the quick increase in the exchanging data size is. Based on this regard, this paper proposes a wired and wireless protocol that applies cooperation Network coding (CoNC) in a wired ring topology (WRT) to improve exchanging the BD significantly in wireless mesh network (WMN). The paper presents a solution for distributed nodes to deal with big data over 5G by proposing Hybrid Ring-Mesh Protocols (HRMP) that exploit the CoNC technique at distributed nodes. The proposed protocol (X-ORING) deterministically combines the data that is received at a base station (BS), where the BS wirelessly retransmits the combined data to the WMN members, instead of just forwarding them to the WMN members. Moreover, all members of the WMN are connected by wired optical fibre channels in a WRT and directly to the BS. The results show that applying CoNC in the proposed protocols exploits the advantages of the WRP between the WMN members, and consequently, the WMN packet error rate is significantly improved. Moreover, using optical fibre wires between the mesh network members and the BS increases the WMN coverage region considerably, and allows the BS to receive all members' packets correctly. Finally, the results show that applying CoNC on the WRT improves the entire network maintenance and reliability greatly, simply because the proposed HRMP can continue broadcasting even if one of the direct optical fibre goes out of serves, i.e. the fibre link between one of the $\mathrm{N}$ member and the BS lost the connectivity.
\end{abstract}

Keywords: Network coding, 5G networks, Wireless mesh network, Big data

\section{Introduction}

Big data (BD) communication networks are needed for almost all modern applications, starting from simple local networks that may consist of several members to complicated satellite and mobile networks that may connect a large number of users. Based on this regard, new research directions are recently being developed to adapt to this high pace of exchanging data size.

Though BD is a widespread and well-used expression, but yet there is no clear identification with specific parameters to identify the $\mathrm{BD}$, so, to have a clear manner when

(c) The Author(s), 2021. Open Access This article is licensed under a Creative Commons Attribution 4.0 International License, which permits use, sharing, adaptation, distribution and reproduction in any medium or format, as long as you give appropriate credit to the original author(s) and the source, provide a link to the Creative Commons licence, and indicate if changes were made. The images or other third party material in this article are included in the article's Creative Commons licence, unless indicated otherwise in a credit line to the material. If material is not included in the article's Creative Commons licence and your intended use is not permitted by statutory regulation or exceeds the permitted use, you will need to obtain permission directly from the copyright holder. To view a copy of this licence, visit http:// creativecommons.org/licenses/by/4.0/. 
dealing with $\mathrm{BD}$, this paper identifies the $\mathrm{BD}$ by the data that is considered big to be processed smoothly and without creating a problem in the network because of its size. Indeed this identification makes the data size be a criteria to deal with BD issues without ignoring the other issues, which means that data size becomes not the only criteria.

The divide-and-conquer method is one of the relatively new techniques that was applied to mitigate the effect of $\mathrm{BD}$, such as in $[1,2]$, where $\mathrm{BD}$ analysis was performed for a cluster of computers.

In [3], BD technical challenges were investigated and hence suggested Divide-andConquer as an effective method to deal with BD applications.

Recently, many research work has been released that investigates BD issues, such as in [4-8], where in [4] the analysis of data partitioning and sampling method is introduced, while [5] provides valuable and recent explains for BD fundamental concepts and analysis according to the theoretical army command information system.

In [6], the analysis of a large-scale data distribution management system was suggested to be tickled by a random sampling of the entire data.

Unlike [1-6], this paper proposes implementing cooperating network coding (CoNC) as a useful technique to help in solving the $\mathrm{BD}$ challenges for mesh network (MN).

CoNC presented in [7-13] as a useful technique to improve the data rate and decrease the need for the Automatic Repeat Request (ARQ), besides improving the Packet error rate (PER). The outcome from [7-13] shows that CoNC is regarded as an excellent technique to decrease the number of ARQ at an acceptable consequence of increasing the bit error rate (BER) in decode-and-re-encode technique, mainly when implementing a good forward error correction code.

Implementing ring topology (RT) in a wireless $\mathrm{MN}$ has presented early in [14], where RT was proposed to improve the mesh architecture fault tolerance, based on requiring the support from eight-neighbourhood members connected to the faulty one, regardless of the network's data size and the number of network's members, unlike of the proposed work, where all nodes of the ring network cooperate together to complete the communication for all members, by the support of a base station (BS).

Later on, Zheng et al. [15] showed how the RT tends to reduce the mechanical load performed in the wireless mesh central node. Still the work applied in [15] neither exploited the benefits of the CoNC, nor adjusted to fit BD communication networks. Moreover, the presented work in the proposed paper investigates the PER over erasure channels to show the practicality of the proposed HRMP for BD communication networks.

The relation between the number of connected members in the RT with the coverage region and network capacity investigated in [16], which clearly declared that implementing RT extends the mesh wireless coverage region. Though [16] managed to extend the mesh coverage region; still it did not fully exploit the advantage of applying RT, unlike the proposed work in this paper, which imposes CoNC on RT and the BS, resulting in enabling the proposed HRMP to enlarge the coverage region and to be more suitable for BD communication networks, as a result of improving the PER.

Recently in [17], a Hybrid Mesh-Ring topology presented for Bluetooth networks, which showed that the optimum Bluetooth configuration could be achieved to 
outperform the conventional ring-based method. Again [17] proved that HRMP has a valuable improvement when applied to particular applications.

The research work in [18-20] introduces useful proposes for applying Network Coding $(\mathrm{NC})$ on wireless $\mathrm{MN}$, which shows how $\mathrm{NC}$ can be helpful to improve the capacity throughput [18], improve the performance of the self-similar flow [19], and improve the multicast acceptance rate for multicast routing in multi-channel multi-radio [20].

Indeed, researches on $\mathrm{MN}$ are mainly directed to increase the region's size, power efficiency, and reliability; for example, in [21], an experimental procedure implements ZigBee to determine the power consumption and reliability optimization, and [22] presented a mobile WMN where an autonomous control method of distribution is applied in a mobile WMN to enhance the energy consumption. More advanced research work performed in [23], where a wireless $\mathrm{MN}$ was proposed for the Internet of things (IoT) systems for smart homes and cities, undeniably [23] showed how WMN could be improved to adapt with such specific applications.

The proposed HRMP in this paper combines the advantages of applying a wired ring topology (WRT), with the advantages of applying a WMN to exchange BD via a BS. Hence, the BS applies CoNC on the received data before broadcasting the network coded data through erasure channels, resulting in improving the PER, reducing the $\mathrm{ARQ}$, increasing the mesh coverage region, and decreasing the number of required wires, taking into consideration that reducing the number of required wires and the relative hardware significantly reduces the establishment and maintenance cost of the network, besides improving the network complexity and reliability as a results of applying CoNC on the WRT network.

The 5G networks and beyond are expected to be able to apply Artificial Intelligence (AI) and Machine Learning (ML) in wireless networks [24], and for unbalanced data in general [25], accordingly, proposing a deterministic fault avoidance protocol is regarded as in important step toward AI and ML fault corrections in wireless networks, such as the deterministic protocols proposed and discussed in [26].

The rest of the paper is organized as follows: Sect. 2 explains the system model proposed in this paper with the relative equations and designs, where Sect. 3 introduces the analytical results through MATLAB software codes to illustrate the benefits of the proposed work. Finally, Sect. 4 concludes the paper and presents the future work.

\section{The system model and proposed method}

Mesh topology is usually implemented in wired networks, where all nodes can exchange their data over direct-wired channels as shown in Fig. 1 for an MN of six members $(M=6)$ as an example. Figure 1 shows that MN suffers from a large number of wired cables with their relative connections' hardware; indeed, for $M$ nodes $\mathrm{MN}, M(M-1)$ cables and their relative hardware are needed, which is the reason of limiting the wired MN coverage region to ten meters or so. Moreover any connection problem between any two members; results in a total disconnection between them till removing the connection trouble. When a BD exchange network is needed for a large coverage communication region, wired $\mathrm{MN}$ becomes practically unsuitable. Consequently, this paper proposes a mix of wired and wireless protocols that exploit CoNC advantages to help in solving the MN challenges for BD networks. Indeed, results 


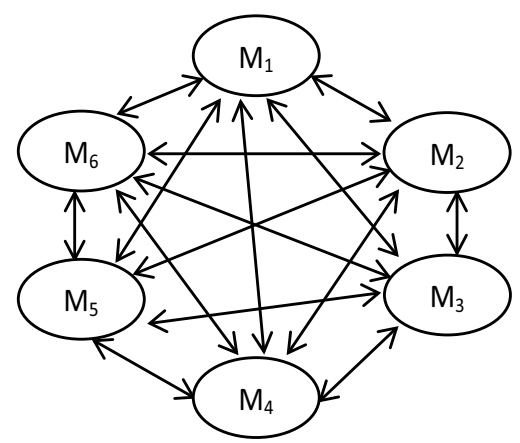

Fig. 1 Wired mesh network for $M=6$ members that are connected by $M(M-1)$ fibre cables

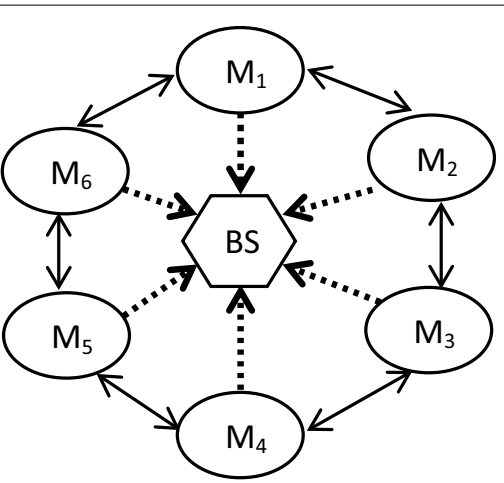

Fig. 2 Six members are connected in a wired ring protocol to each other (solid lines) and a BS (dashed lines)

show that the proposed protocols significantly increase the MN coverage region and improve the MN applicability, as results, the proposed protocols assist in increasing the number of connected members and simplifying the algorithm that could be adopted for BD in $5 \mathrm{G}$ networks.

\subsection{The proposed hybrid ring-mesh benchmark}

In the HRMP Benchmark scenario, $M$ members are connected to each other's by a WRT that requires $M$ cables and their relative hardware elements. Simultaneously, the $M$ members are connected to a BS by $M$ optical fibre wires, consequently, $2 M$ wires are required in the proposed Benchmark scenario.

The $M$ members are connected to exchange their data through the WMN, where each member aims to receive and then retrieve the other $M-1$ partner's information, as shown in Fig. 2 for a network of six members as an example.

Figure 2 shows that only twelve $(2 M)$ wires are needed for this network because the BS is broadcasting the $M$ member's data wirelessly.

In such a scenario where CoNC is not applied, the BS forwards the received $M$ packets for the $M$ members. After completing a full successful transmission stage, each member will be receiving the other $M-1$ packets from the BS, besides its neighbour's two packets through the ring connected wired topology. It is important to 
notice that the BS broadcasts the received $M$ packets separately, i.e. it does not combine (X-ORING) packets. The broadcast packets in the Benchmark scenario are called in this paper "single packets."

Accordingly, the received matrix for node four-as an example-is shown in Eq. 1:

$$
\operatorname{Rp}_{(M 4)}=\left[\begin{array}{llllll}
M_{1} & M_{2} & M_{3} & M_{4} & M_{5} & M_{6}
\end{array}\right]\left[\begin{array}{llllll}
1 & 0 & 0 & 0 & 0 & 0 \\
0 & 1 & 0 & 0 & 0 & 0 \\
0 & 0 & 1 & 0 & 0 & 0 \\
0 & 0 & 0 & 1 & 0 & 0 \\
0 & 0 & 0 & 0 & 1 & 0 \\
0 & 0 & 0 & 0 & 0 & 1
\end{array}\right] \text { and }\left[M_{3} M_{5}\right]\left[\begin{array}{llllll}
0 & 0 & 1 & 0 & 0 & 0 \\
0 & 0 & 0 & 0 & 1 & 0
\end{array}\right]
$$

where $\mathrm{Rp}_{(\mathrm{M} 4)}$ denotes to the single received packets at the fourth member.

According to Eq. 1, the $M_{4}$ member receives a maximum of five single packets from the $\mathrm{BS}$ and two single packets from its neighbour members, i.e. $M_{3}$ and $M_{5}$, besides the fact that $M_{4}$ knows its packet, accordingly, the retrieving matrix at $M_{4}$ when all packets are assumed to be received correctly, is:

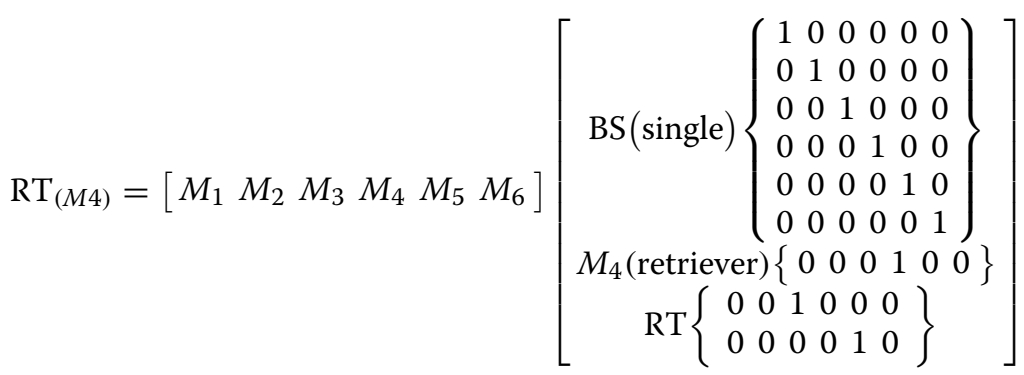

where $\mathrm{RT}_{(\mathrm{M} 4)}$ denotes to the retrieving matrix at $M_{4}$ and $\mathrm{BS}$ (single) denotes to the single packets received from the BS (without applying CoNC).

When taking into consideration that the duplicated packets (received twice) is regarded as bandwidth and power waste ones; because they do not improve the retrieving matrix's rank (Eq. 2) as they are not new linearly dependant equations, hence the RT and the retriever's packet are considered as redundant.

Accordingly, Eq. 2 shows that the packets received through the WRT do not have any new information, and it is the same for each member's packet in the whole network; so these three packets are pure redundant and hence discarded, which is considered as loss of power and more traffic for no new information.

The Jordan Gaussian Elimination (JGE) retrieving mechanism confirms that a receiver can retrieve all received packets when and only when the received retrieving matrix has a rank that equals the number of the unknown pivots $[8,11]$. Based on JGE retrieving condition, each member of the WMN can retrieve all other members if the received rank of Eq. 2 equals $M$.

In the case that the BS drops any number of packets through the assumed lossy channels between all members and $M_{4}$, -as an example-the communication will not be fully completed unless if the dropped packets are the packets that were sent for RT packets (neighbours) $\left(M_{3}\right.$ or/and $\left.M_{5}\right)$ or the own retriever packet $\left(M_{4}\right)$. On the other hand, if the retriever member does not receive any packet other than the three known packets (RT and retriever), the communication will not be completed, 
i.e. the retrieving matrix's rank (Eq. 2) will be less than $M$. Based on the above, the RT improved the WMN partially, and when assuming a large number of members, such as tens members, this improvement becomes trivial, as shown in the result Sect. 3 .

The only remaining advantage of the Benchmark scenario is the ability of the network to enlarge the coverage region of the mesh network. Yet, the severe relation between the transmission distance and the PER in lossy network channels makes the coverage region to be limited even in the existence of the WRT. Moreover, if any disconnection problem happens to any optical fibre that connects any member of the $M$ WMN members to the BS, the communication will not be completed for the disconnected member because its packet will not be received at the BS, and hence will not be broadcast to the rest of members, just only this member's neighbours that can receive the packet through the wired RT.

Network reliability of the Benchmark scenario is clearly at the minimum level, i.e. any maintenance over any optical fibre for the WNM results in isolating the optical fibre's member from sending its data to the BS, as shown in Fig. $3 \mathrm{a}$ for $M_{4}$ as an example. Moreover, if any disconnection happens between any two neighbours and either neighbours with the BS, the communication between these two neighbours will be disconnected and at the same time, the connection between either member with the BS is lost; consequently, the full communication between them will be lost, though they are neighbours, as shown in Fig. $3 \mathrm{~b}$ for $M_{1}$ and $M_{2}$ as an example.

Figure 3a shows the case when a wire between an MN member and the BS is disconnected and Fig. 3b shows the case when a wire between two neighbours is disconnected simultaneously with a wire between the BS and either neighbour of the disconnected wire to the BS ( $M_{1}$ to BS is proposed). Accordingly, in Fig. 3a, $M_{4}$ is isolated except from its two neighbours, where in Fig. $3 \mathrm{~b}, M_{1}$ 's information will not be received at $\mathrm{M}_{2}$ though they are neighbours.

Based on the above, the Benchmark scenario does not provide significant benefits to the whole network, other than improving the coverage region that severely depends on the PER in the channels between the N WMN members.

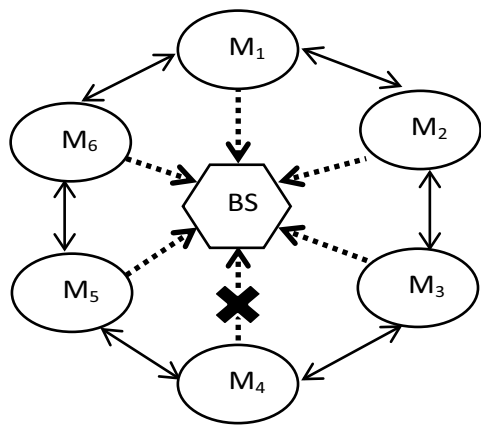

(a)

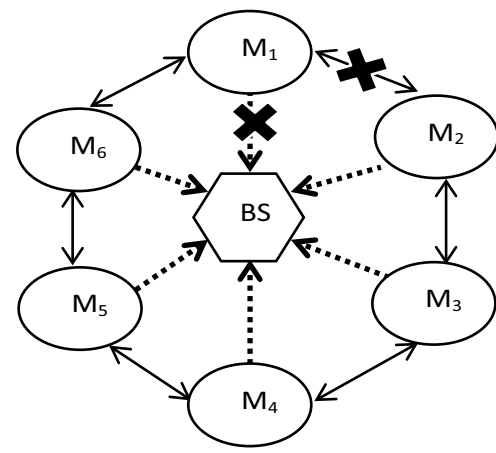

(b)

Fig. 3 Faults in the wired connections that result in the failure of the full communication 


\subsection{The proposed hybrid ring-mesh cooperation network coding protocol}

Based on the disadvantages in the Benchmark scenario mentioned in Sect. 2.1, CoNC is proposed to fully exploit the benefits of the HRM connection shown in Fig. 2. Indeed, if the CoNC applied to the BS in a deterministic manner, the WRT plays such a significant role to improve the full connectivity of the $\mathrm{MN}$, in addition to the original benefit of increasing the WMN coverage region, taking into consideration that CoNC mitigates the effect of the PER significantly [8], and hence improves the coverage region.

The proposed protocol is as follows: the BS manipulates the $M$ single packets received from the optical fibre channels by deterministically combining (X-ORING) $M-1$ different packets for $M$ times, where every single packet is excluded just once from the $M$ combination times, resulting in $M$ different combined packets. This combination algorithm is called in this paper as " $M-1$ combination". Accordingly, the $M$ deterministically combined packets, which are named "Network Coded Packets" (NCP), are as in Eq. 3 for a WMN of six members as an example:

$$
T_{\mathrm{NCP}}=\left[\begin{array}{llllll}
M_{1} & M_{2} & M_{3} & M_{4} & M_{5} & M_{6}
\end{array}\right]\left[\begin{array}{llllll}
0 & 1 & 1 & 1 & 1 & 1 \\
1 & 0 & 1 & 1 & 1 & 1 \\
1 & 1 & 0 & 1 & 1 & 1 \\
1 & 1 & 1 & 0 & 1 & 1 \\
1 & 1 & 1 & 1 & 0 & 1 \\
1 & 1 & 1 & 1 & 1 & 0
\end{array}\right]
$$

where $T_{\mathrm{NCP}}$ denotes to the created NCP packets that are transmitted from the BS to the $M$ members of the WMN, taking into consideration that the BS receives correctly the $M$ single packets from the $M$ WMN members through the optical fibre channels, so, it is always assumed that the $\mathrm{BS}$ receives the $M$ packets correctly in a single form, i.e. each packet contains data for just one single member.

The received packets from the BS and $\mathrm{RTat} \mathrm{M}_{4}$, for example, are shown in Eq. 4:

$$
\operatorname{Rp}_{(M 4)}=\left[\begin{array}{llllll}
M_{1} & M_{2} & M_{3} & M_{4} & M_{5} & M_{6}
\end{array}\right]\left[\begin{array}{llllll}
0 & 1 & 1 & 1 & 1 & 1 \\
1 & 0 & 1 & 1 & 1 & 1 \\
1 & 1 & 0 & 1 & 1 & 1 \\
1 & 1 & 1 & 0 & 1 & 1 \\
1 & 1 & 1 & 1 & 0 & 1 \\
1 & 1 & 1 & 1 & 1 & 0
\end{array}\right] \text { and }\left[\begin{array}{ll}
M_{3} & M_{5}
\end{array}\right]\left[\begin{array}{llllll}
0 & 0 & 1 & 0 & 0 & 0 \\
0 & 0 & 0 & 0 & 1 & 0
\end{array}\right]
$$

where $\mathrm{RT}_{(\mathrm{M} 4)}$ denotes to the received packets at $M_{4}$, noticing that the receiving row for any none received packet will be a row of zeroes in Eq. 4.

Considering that $M_{4}$ knows its packet, so, the JGE retrieving matrix at $M_{4}$ is given in Eq. 5:

$$
\mathrm{RT}_{(M 4)}=\left[\begin{array}{llllll}
M_{1} & M_{2} & M_{3} & M_{4} & M_{5} & M_{6}
\end{array}\right]\left[\begin{array}{llllll}
0 & 1 & 1 & 1 & 1 & 1 \\
1 & 0 & 1 & 1 & 1 & 1 \\
1 & 1 & 0 & 1 & 1 & 1 \\
1 & 1 & 1 & 0 & 1 & 1 \\
1 & 1 & 1 & 1 & 0 & 1 \\
1 & 1 & 1 & 1 & 1 & 0
\end{array}\right] \text { and }\left[\begin{array}{lll}
M_{3} & M_{4} M_{5}
\end{array}\right]\left[\begin{array}{llllll}
0 & 0 & 1 & 0 & 0 & 0 \\
0 & 0 & 0 & 1 & 0 & 0 \\
0 & 0 & 0 & 0 & 1 & 0
\end{array}\right]
$$

According to Eq. 5, the retrieving matrix is shown in Eq. 6. 


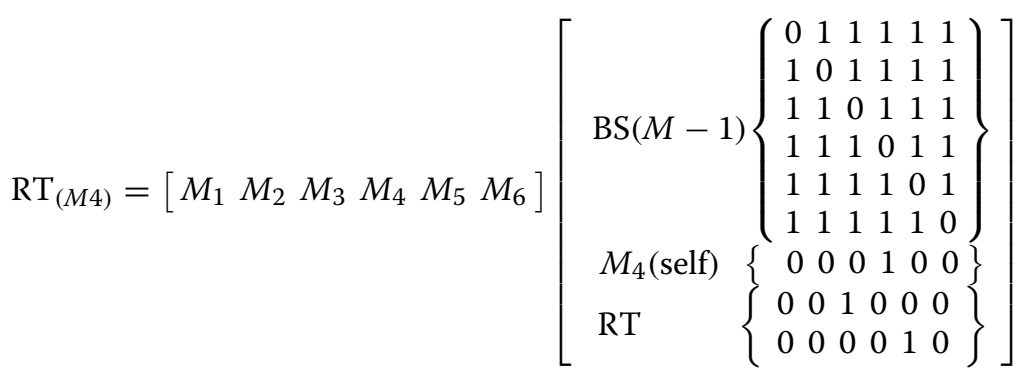

where $\mathrm{RT}_{(\mathrm{M} 4)}$ denotes to the retrieving matrix for $M_{4}$, as an example.

Equation 6 confirms that after the transmission processes, each member of the $M$ WMN is expected to receive $M \mathrm{NCP}$, besides the fact that each member knows its packet and its two neighbours' as well, accordingly the two packets received by the WRT and the member's packet are regarded as three new packets with novel information, based on in Eq. 6.

Though three new novel packets seem to be a small number of packets when compared to a network of tens of members, the effect of these three packets is significant, simply because each member becomes able to retrieve its data even when losing any three packets transmitted from the BS, which is unlike the Benchmark scenario, where each member cannot complete the communication when losing any packet other than its two neighbours.

The reason that each member can retrieve the information for all other members even when not receiving three packets has become dropping any three NCPs from the proposed deterministically combined packets on the BS still gives a transmission matrix of rank $M$ in Eq. 6. The rank of Eq. 6 remains equal $M$ because each single packet (from RT) gives new information to the transmission matrix, so the reception matrix rank remains equal $M$ when replacing the three single packets with any three combined packets in the received matrix (Eq. 6).

GJE processes theory confirms that any matrix of $\operatorname{rank} M$ that contains $M$ pivots can be retrieved successfully when receiving $M$ linearly independent equations, regardless these equations are for single or combined packets $[8,11]$. Accordingly, the CoNC enables each member to drop any three packets through the lossy wireless channel between the $M$ WMN members, which is such an important advantage that helps in increasing the coverage region and decreasing the ARQ significantly.

The advantage of applying the deterministic combination to the BS can be extended by creating more linearly independent combined packets to produce additional new novel information, as shown in [9].

To enhance the full reception for each member of the WMN, the BS can create more than $M$ novel (linearly independent) combined packets to assure that there is no any created packet that does not maintain the $\operatorname{rank} M$ of the receiving matrix when replaced with the lost packet.

Based on the network data rate, erasure probability of the WMN channel, and the required coverage region; the $\mathrm{BS}$ can create as many novel combinations as required, taking into consideration that the novel packet is regarded as the packet that can be replaced in Eqs. 2 and 6 without decreasing the matrix's rank (rank=M). 
So, it is clear that implementing WRN with applying CoNC on the BS gives an important trade-off between the data rate and both of the PER and the coverage region, which is investigated through the simulation results in Sect. 3.

Several deterministic combinations can maintain the full rank of the receiving matrix when replaced with the lost packets, which were proposed in [9] for different scenarios, such as Odd-Even combination, Next-Neighbour combination, PreviousNeighbour combination, and others. The key issue is to follow a deterministic manner of combination to assure that the same NCP is not created and broadcast more than once to avoid a redundant transmission that does not improve the retrieving matrix's rank.

It is easy to notice that when combining all of the $M$ packets, a new novel packet is created, so the BS can broadcast $M+1$ packets to enable each member to drop even four different packets, as shown in Eq. 7

$$
\operatorname{RT}_{(M 4)+1}=\left[\begin{array}{llllll}
M_{1} & M_{2} & M_{3} & M_{4} & M_{5} & M_{6}
\end{array}\right]\left[\begin{array}{c}
\mathrm{BS}(M-1)\left\{\begin{array}{llllll}
0 & 1 & 1 & 1 & 1 & 1 \\
1 & 0 & 1 & 1 & 1 & 1 \\
1 & 1 & 0 & 1 & 1 & 1 \\
1 & 1 & 1 & 0 & 1 & 1 \\
1 & 1 & 1 & 1 & 0 & 1 \\
1 & 1 & 1 & 1 & 1 & 0
\end{array}\right\} \\
M_{4}(\text { retriever })\left\{\begin{array}{lllllll}
0 & 0 & 0 & 1 & 0 & 0
\end{array}\right\} \\
\mathrm{RT}\left\{\begin{array}{lllllll}
0 & 0 & 1 & 0 & 0 & 0 \\
0 & 0 & 0 & 0 & 1 & 0
\end{array}\right\} \\
\text { All combined } \left.\begin{array}{llllll}
1 & 1 & 1 & 1 & 1 & 1
\end{array}\right\}
\end{array}\right]
$$

where $\mathrm{RT}_{(M 4)+1}$ is the retrieving matrix at $M_{4}$ with the extra suggested all combined packet in the case of full reception.

The Odd-Even combination can create $M$ new novel (linearly independent) combination protocol [9], which is one of the recommended protocols for this scenario.

Equation 8 shows the retrieving matrix for the $M$ created novel packets when the Odd-Even combination algorithm is adopted at the BS.

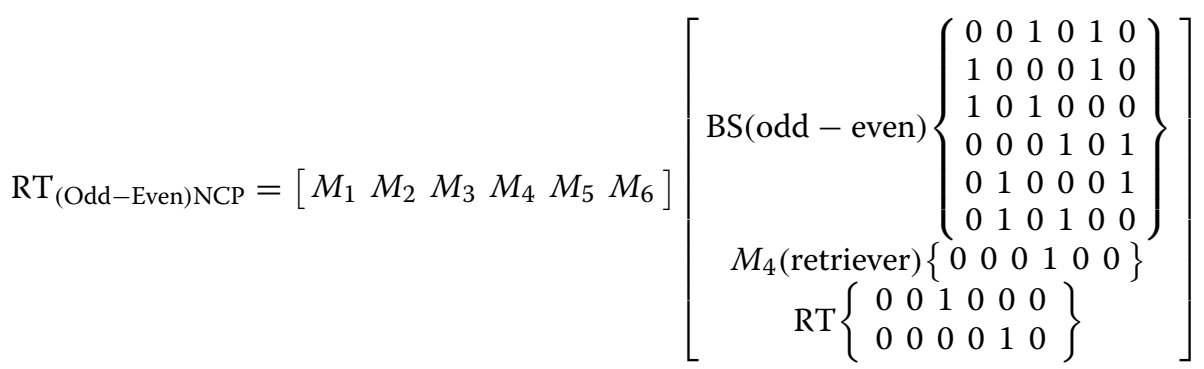

where $\mathrm{RT}_{\text {(Odd-Even)NCP }}$ is the retrieving matrix at $M_{4}$ as an example when the Odd-Even combined packets algorithm is applied.

The deterministic Odd-Even combination process is partially similar to the combination proposed in Eq. 2; just it is applied twice, once for even members where all even members are combined excluding each even packet just once, and the other time when odd members are combined excluding each odd packet just once, resulting to $M$ new linearly independent equations as shown in Eq. 8. 
Other deterministic combination algorithms can be created, such as Eq. 9, where the Next-Neighbour combination algorithm is adopted, and the Previous-Neighbour combination algorithm is shown in Eq. 10, where each packet is combined just with the previous neighbour's packet.

$$
\mathrm{RT}_{(\mathrm{NN}) \mathrm{NCP}}=\left[\begin{array}{llllll}
M_{1} & M_{2} & M_{3} & M_{4} & M_{5} & M_{6}
\end{array}\right]\left[\begin{array}{c}
\mathrm{BS}(\mathrm{NN})\left\{\begin{array}{llllll}
1 & 1 & 0 & 0 & 0 & 0 \\
0 & 1 & 1 & 0 & 0 & 0 \\
0 & 0 & 1 & 1 & 0 & 0 \\
0 & 0 & 0 & 1 & 1 & 0 \\
0 & 0 & 0 & 0 & 1 & 1 \\
1 & 0 & 0 & 0 & 0 & 1
\end{array}\right\} \\
\left.M_{4} \text { (retriever) } \begin{array}{llllll}
0 & 0 & 0 & 1 & 0 & 0
\end{array}\right\} \\
\mathrm{RT}\left\{\begin{array}{llllll}
0 & 0 & 1 & 0 & 0 & 0 \\
0 & 0 & 0 & 0 & 1 & 0
\end{array}\right\}
\end{array}\right]
$$

where $\mathrm{RT}_{(\mathrm{NN}) \mathrm{NCP}}$ is the retrieving matrix for the Next-Neighbour combination algorithm at $M_{4}$ as an example.

$$
\operatorname{RT}_{(\mathrm{PN}) N C P}=\left[\begin{array}{llllll}
M_{1} & M_{2} & M_{3} & M_{4} & M_{5} & M_{6}
\end{array}\right]\left[\begin{array}{c}
\mathrm{BS}(\mathrm{PN}) \\
\left.\begin{array}{llllll}
1 & 0 & 0 & 0 & 0 & 1 \\
1 & 1 & 0 & 0 & 0 & 0 \\
0 & 1 & 1 & 0 & 0 & 0 \\
0 & 0 & 1 & 1 & 0 & 0 \\
0 & 0 & 0 & 1 & 1 & 0 \\
1 & 0 & 0 & 0 & 1 & 1
\end{array}\right\} \\
\left.M_{4}(\text { retriever }) \begin{array}{llllll}
0 & 0 & 0 & 1 & 0 & 0
\end{array}\right\} \\
\operatorname{RT}\left\{\begin{array}{llllll}
0 & 0 & 1 & 0 & 0 & 0 \\
0 & 0 & 0 & 0 & 1 & 0
\end{array}\right\}
\end{array}\right]
$$

where $\mathrm{RT}_{(\mathrm{PN}) \mathrm{NCP}}$ is the retrieving matrix for the Previous-Neighbour combination algorithm at $M_{4}$ as an example.

Any two combinations, such as the $M-1$ combination algorithm and the Odd-Even combination algorithm shown in Eq. 11 can be applied at the BS to generate extra $M$ linearly independent equations.

$$
\begin{aligned}
& \mathrm{RT}_{(\text {Odd-Even and } M-1) \mathrm{NCP}}
\end{aligned}
$$

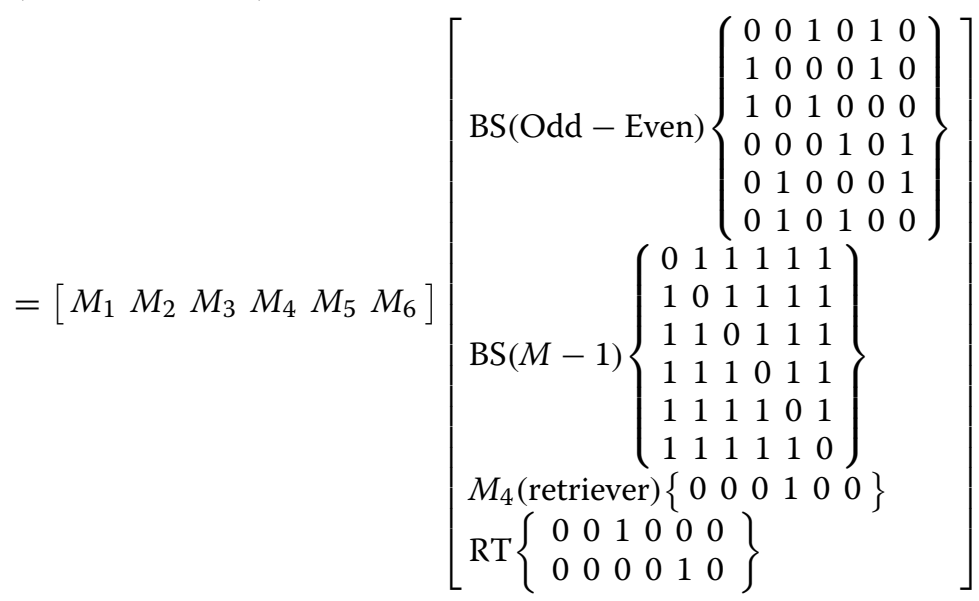

where $\mathrm{RT}_{(\text {Odd-Even and } N-1) \mathrm{NCP}}$ is the retrieving matrix for the Odd-Even and the $M-1$ combination algorithms at $M_{4}$ as an example. 
According to Eq. (11), the BS broadcasts $2 M$ packets instead of $M$, which is regarded as a trade-off between the data rate and the PER, considering that the better PER, the larger the WMN coverage region.

So, when sending $2 M$ novel packets, besides the pre-mentioned three packets (RT and retriever's packet), each wireless member's channel has the permeability to drop till $M+3$ packets, which is regarded as an extremely great improvement to the WMN PER that enables to increase remarkably the coverage region. Any extra broadcast packet can be set according to the network's PER and the desired coverage region, shown through the simulation results in Sect. 3. For BD transmission, the authors of this paper recommends following fair trade-off between the data rate and the PER (coverage region) so, $M+20 \%$ of $M$ is considered as a reasonable trade-off. However, the extra number of packets depends on the needed coverage region and the PER in the WMN channels. The recommendation mentioned above is based on the authors' personal experience in this field. Still the main rule is as follows: the more transmitted packets, the better performance, coverage region and worse data rate efficiency. Finally, Eqs. 1-11 show how the proposed protocol works in a clear and systematic manner.

\subsection{Cooperation networking coding over ring topology and reliability improvement}

When a wire that connects a member with the BS got disconnected as a result of maintenance operations, or any an unexpected fault as shown in Fig. 3a, or two wires got disconnected as shown in Fig. 3b, the disconnected member is named in this paper as a "dead member"; however, CoNC can solve this problem once applied over the RT in the following algorithm:

The data packet of the dead member already sent to the two neighbours; hence, each dead member's neighbour combines (X-ORING) the dead packet's data to its data packet and then sends the combined packet to the BS through its wires. Consequently, the dead member's neighbours pass the dead member's data packet to their neighbours instead of passing their data packets. The rest of the WMN members do the same, i.e. combining the dead member's data packet to their packets and passing the dead member's data packet to their neighbours, as shown in Fig. 4a, b.

At the end of the passing processing shown in Fig. 4a, b, each member of the WMN will be sending its data combined with the dead member's data. Accordingly, the BS will be receiving Eq. 12 when assuming that $M_{4}$ is the dead member as in Fig. $4 \mathrm{a}$, and Eq. 13 if $M_{1}$ got two disconnected wires as shown in Fig. 4b:

$$
\operatorname{Rp}_{(\mathrm{BS})=}\left[\begin{array}{llllll}
M_{1} & M_{2} & M_{3} & M_{4} & M_{5} & M_{6}
\end{array}\right]\left[\begin{array}{llllll}
1 & 0 & 0 & 1 & 0 & 0 \\
0 & 1 & 0 & 1 & 0 & 0 \\
0 & 0 & 1 & 1 & 0 & 0 \\
0 & 0 & 0 & 0 & 0 & 0 \\
0 & 0 & 0 & 1 & 1 & 0 \\
0 & 0 & 0 & 1 & 0 & 1
\end{array}\right]
$$

where $\mathrm{Rp}(\mathrm{BS})$ denotes to the received packets at the $\mathrm{BS}$ that have been sent through the $M-1$ wires based on Fig. 4a.

Equation 12 shows that the BS receives five combined packets $(M-1)$, and the row of the disconnected wire (row 4 in Eq. 12 in this example) is zeroed. 


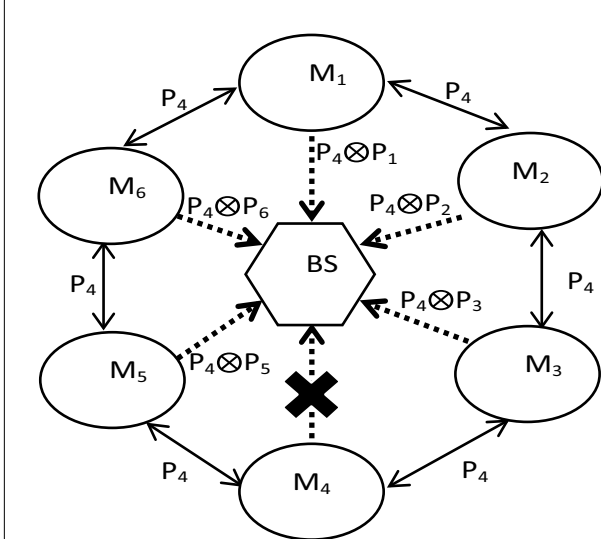

(a)

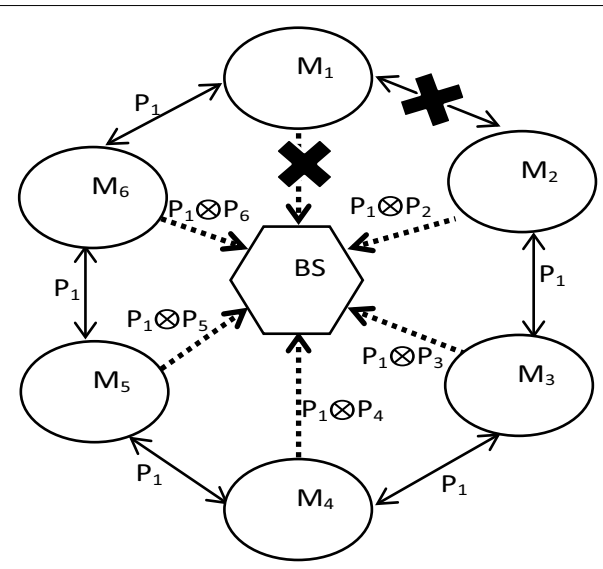

(b)

Fig.4 The scenario where full communication is obtained with faulty connections

$$
\mathrm{Rp}_{(\mathrm{BS})=}\left[\begin{array}{llllll}
M_{1} & M_{2} & M_{3} & M_{4} & M_{5} & M_{6}
\end{array}\right]\left[\begin{array}{llllll}
0 & 0 & 0 & 0 & 0 & 0 \\
1 & 1 & 0 & 0 & 0 & 0 \\
1 & 0 & 1 & 0 & 0 & 0 \\
1 & 0 & 0 & 1 & 0 & 0 \\
1 & 0 & 0 & 0 & 1 & 0 \\
1 & 0 & 0 & 0 & 0 & 1
\end{array}\right]
$$

Equation 13 shows that row one is zeroed because $M_{1}$ is the dead member; hence, the way to retrieve the all member's data is similar to the case when one wire is disconnected.

In the case of full and correct WMN broadcasting, each member of the $M$ WMN will be receiving the same $M-1$ combined packets, including the dead member. The retrieving processes for each member simply performed by X-ORING the retriever's packet with each combined packet separately, to retrieve the $M-1$ neighbours one by one. Based on the above, applying CoNC enables the network to communicate even if one member is not connected to the BS, which means that the communication can be running in the case of fault or maintenance operations, i.e. the network's reliability is improved.

Based on above, the importance of mixing ring and mesh protocols is justified by the dramatically increasing in the wireless transmission rejoin for some of the resent wireless broadcasting elements, such as Sigfox that can cover a region from 10 to $40 \mathrm{kM}$, LoRa that can cover a region from 5 to $20 \mathrm{kM}$, NB-IoT that can cover a region from 1 to $10 \mathrm{kM}$, LTE-M that can cover a region up to $5 \mathrm{kM}$ radius, and Zegbi that can cover around $200 \mathrm{~m}$ radius region.

Accordingly, the proposed protocols are clearly useful for many practical applications such as, IoT applications, remote sensing applications, smart agriculture, smart cities, and etc.

\section{Simulation results}

The simulation results have been obtained by MATLAB software, where a minimum of 100 errors were collected. The error is identified according to JGE as a received retrieving matrix at $M_{4}$ (proposed receiver example) with a rank less than $M$, where $M$ is the number of the mesh network members. 
Moreover, the collected results are only for user four $(M=4)$, so it is important to declare that the achieved PER gain for the $M \mathrm{MN}$ is $M$ folder times more than the gained improvement at $M_{4}$ when the gain extended for to the whole MN members assuming that each member is expected to gain the same improvement as for $M_{4}$.

The investigation starts from comparing the Benchmark scenario that applies the selfish mode (without combination) for a single stage on the BS, with and without RT, to evaluate the benefits of implementing the RT over the PER.

So, for the Benchmark scenario, Fig. 5 shows that the PER gain resulted from the existence of RT is trivial for a member of twenty MN members, and this is justified by the fact that the extra three packets added from the RT have no new information, accordingly, the retrieving matrix's rank at $M_{4}$ is not improved as a result of these three transmitted packets, as shown in Eq. 2. Indeed, this is regarded as a big loss in terms of the PER improvement that could have been obtained. So, RT is just restricted to improve the WMN coverage region [16].

Moreover, Fig. 5 shows the excellent PER improvement achieved when the BS applies the CoNC rather than broadcasting the single received packets from the optical fibre wires in a selfish mode. The Odd-Even protocol outperforms the Benchmark scenario in two to three folders at PER of 0.1, which means that the proposed CoNC protocol exploits the existence of RT in such desired scale. The improvement in the PER becomes much higher when the PER drops to less than 0.1, taking into consideration that the whole WMN will be improved $M$ folders and this is because each member on the WMN behaves similarly to $M_{4}$.

The justification for the good improvement is that the three RP packets give novel information for the full retrieving matrix at $M_{4}$ as an example, which enables $M_{4}$ to drop till three packets before the retrieving matrix's rank becomes less than $M$. the retrieving matrix at $M_{4}$ becomes less than $M$, only when $M_{4}$ drops more than three packets. Similarly, each member of the WMN has the ability to drop up to three packets before its retrieving matrix's rank becomes less than $M$ as shown in Eqs. 6, 8, 9, and 10 for the $M-1$, the Odd-Even, the Next-Neighbour, and the Previous-Neighbour, respectively.

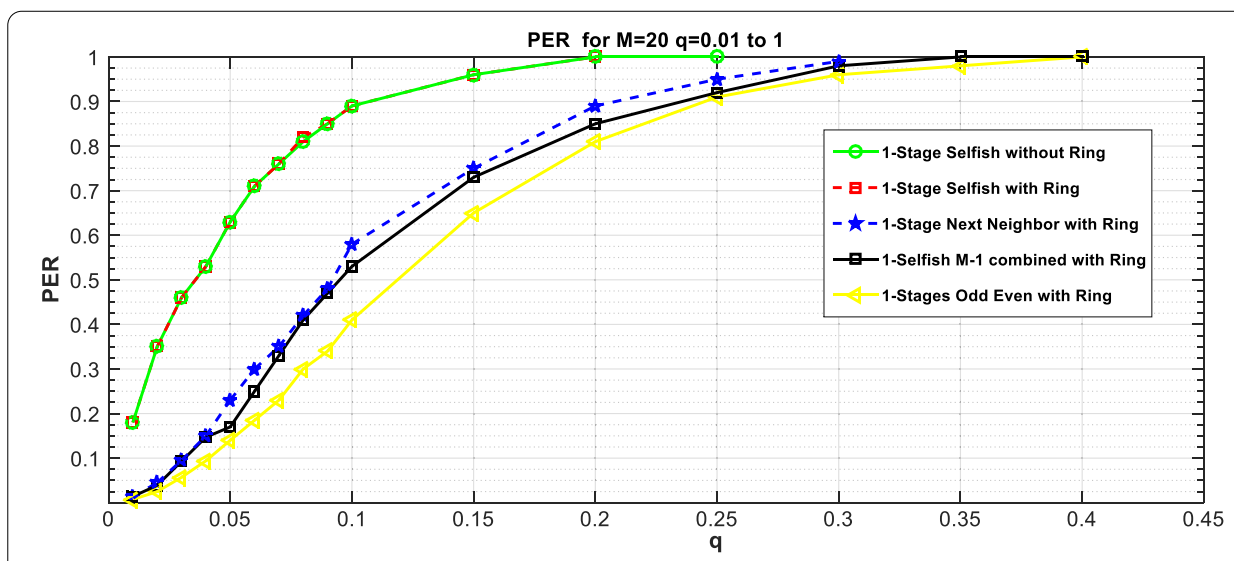

Fig.5 PER for $M=20$ on an HRMP for single stage, when the selfish, the Next-Neighbour, the $M-1$, and the Odd-Even protocols are applied on the BS 
Figure 5 illustrates as well that the Odd-Even combination protocol is the best-proposed one when compared with the Next-Neighbour, and the $M-1$ protocols, which is because the combination algorithm of the Odd-Even has a good diversity of combined packets.

Figure 6 shows the effect of increasing the number of WMN members, starting from ten to twenty. Indeed, Fig. 6 discloses clearly that the PER increases when the number of members increases, and this is because the effect of the three RT packets becomes less effective, i.e. extra three novel packets to a total of ten linearly independent equations, have better influence than three to twenty, simply because it is more likely to drop four packets out of twenty rather than four packets out of ten. (Four lost packets mean that the rank is less than $M$; hence, it is regarded as an error.)

Figure 7 illustrates the big improvement in the PER due to sending an extra one full stage of $M$ combined packets (CoNC packets). The results compare the proposed combination algorithms in the second stage for several scenarios, taking into consideration that two stages of single packets with the RT packets are shown in Fig. 6 for the propose of comparison with the confirmed Benchmark scenario. So, Fig. 7 proves that the effect of the three RT packets has significant improvement. Moreover, the two stages with different combination algorithms in each stage give better PER improvement, which is justified by the more linearly independent equations that results in better retrieving matrix rank. So, there is a valuable improvement when selfish and Odd-Even packets are broadcast by the BS; indeed, the PER improved to 0.04 at the channel erasure probability of 0.1 , compared to 0.1 PER when sending the same single-stage twice. The same improvement can be noticed with the other combination algorithms; however-as in a single-stage-the Odd-Even combination outperforms the other algorithms because of its wide combination diversity for the combined packets.

Figure 8 illustrates the trade-off between sending extra packets and the PER improvement to evaluate the benefits of sending extra packets. The results in Fig. 8 show that the PER can be significantly improved but at the cost of the data rate. Moreover, broadcasting the Next-Neighbour protocol with the Odd-Even gives a better improvement than the $M-1$ combination algorithm.

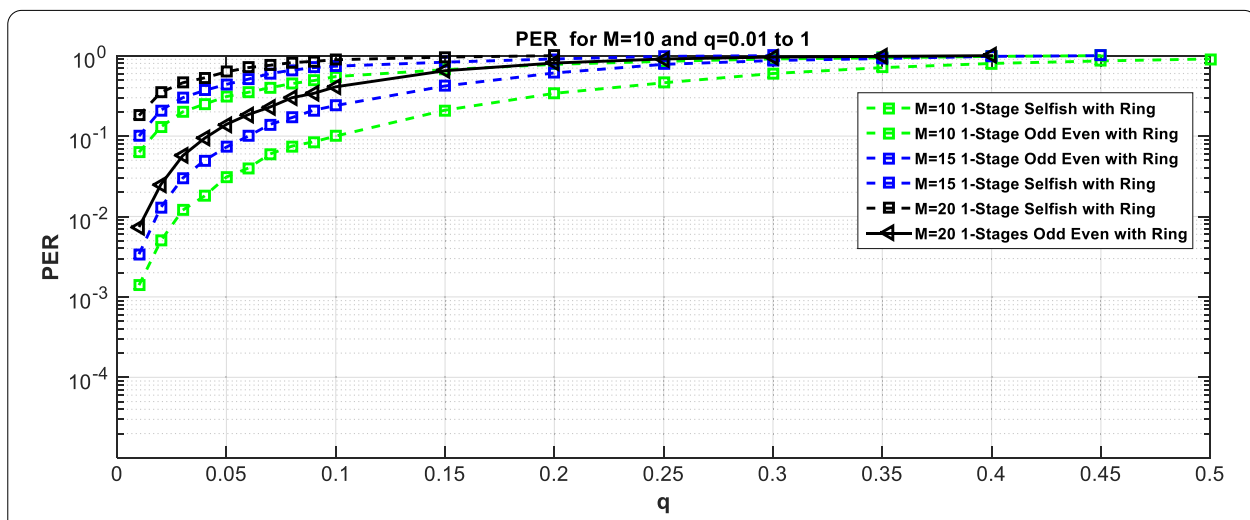

Fig. 6 PER when increasing the number of HRMP members from $M=10$ to $M=20$, for selfish, Next-Neighbour, $M-1$, and Odd-Even protocols 


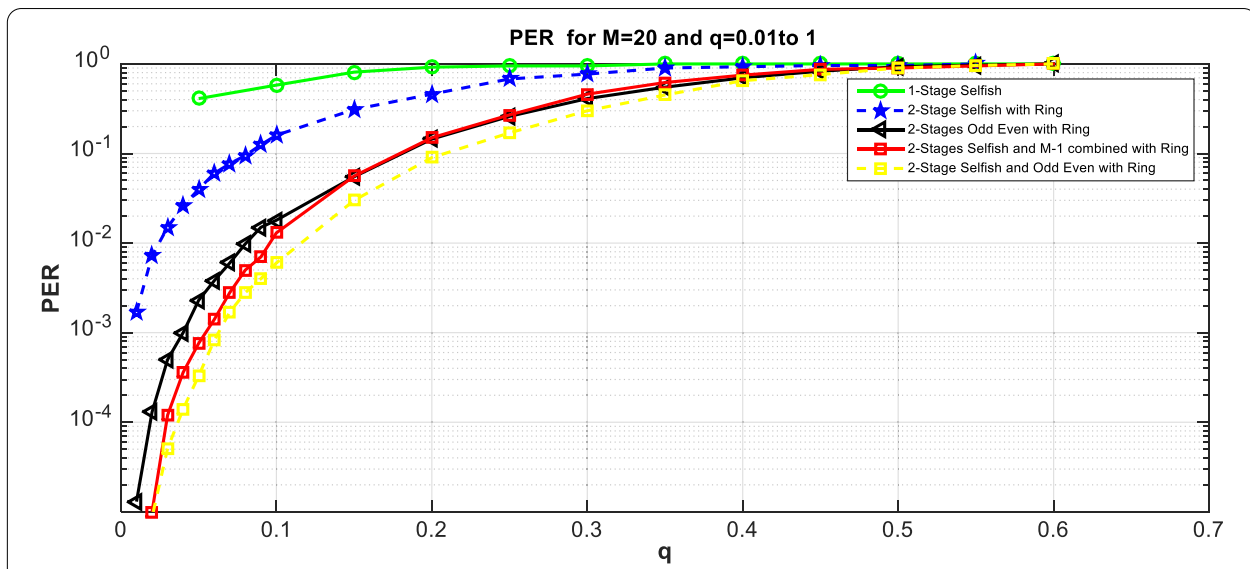

Fig. 7 2-Stage transmission for 2-selfish, 2-Odd-Even, selfish and Odd-Even, and selfish with $M-1$ algorithms

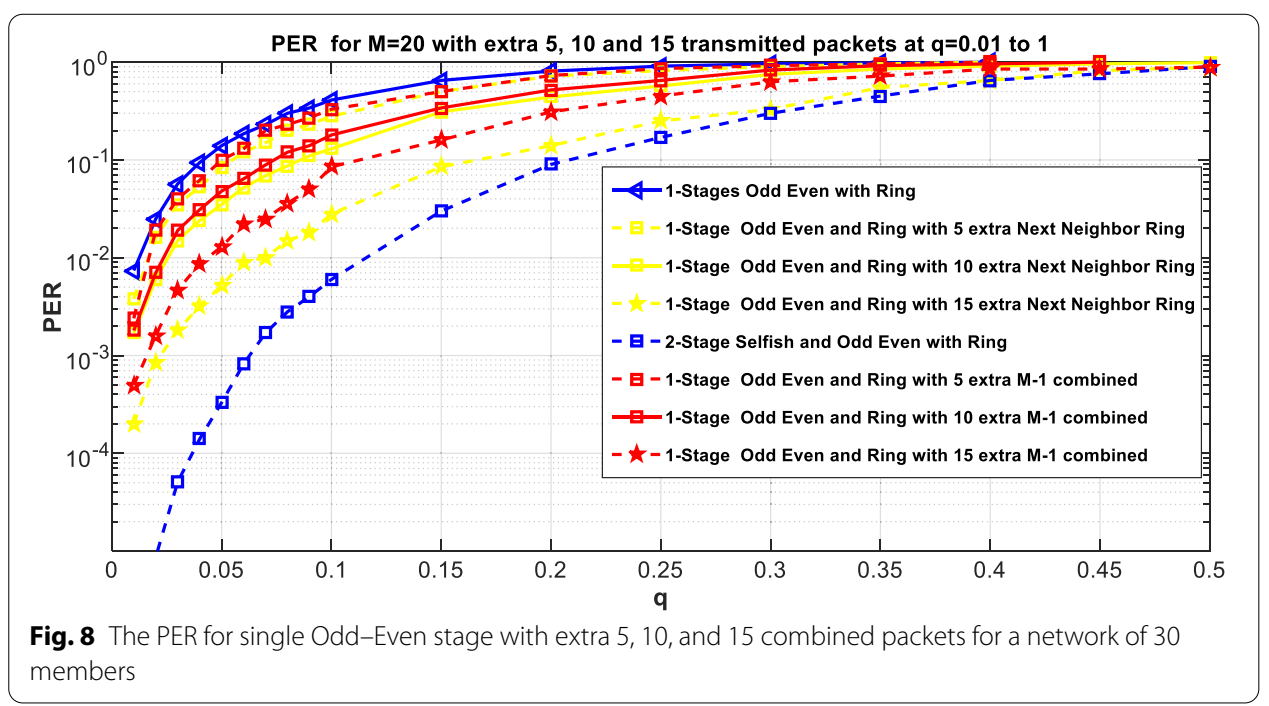

Finally, it is important to mention that processing time for JGE retrieving matrix depends on the number of combined packets, so the fewer combined packets, the shorter the processing time [26], which is the reason to recommend the Odd-Even algorithm rather than the $M-1$ combination, besides to the better PER.

Regarding the reliability improvement achieved from applying CoNC over the RT, Fig. 9 shows that even when one optical fibre wire is not connected between the RT and the BS, as in Fig. 4a and Eq. 12, the communication can continue with better PER than selfish mode. Moreover, when two connections are lost, as in Fig. 4b and Eq. 13, the communication will continue with even better PER than when one optical fibre is disconnected, and this is justified by the strong cooperation algorithm proposed for this scenario where all RP members combine the disconnected member's packet to their packets, resulting to more processing time with better PER. 


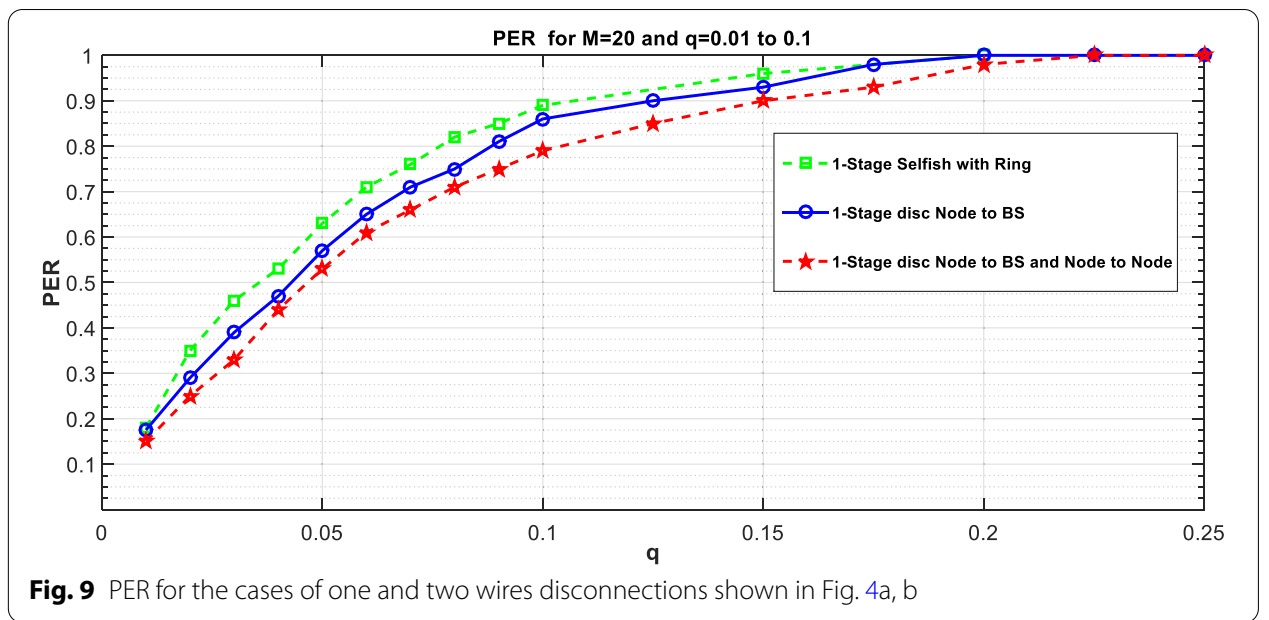

At the end, it is of interest to be mentioned that our research in the physical layer alongside other approaches in the area of 5G networks [27-30] (including high-performance computing for $5 \mathrm{G}$-assisted $\mathrm{IoT} / \mathrm{IoV}$, networking architecture (e.g. software defined networks), big data processing/transmission over 5G, and various $5 \mathrm{G}$ applications (e.g. intelligent transportation systems) [27-33]) can make this topic more interesting for the prospective readers and investigators.

\section{Conclusions}

This paper proposes cooperative network coding (CoNC) algorithms to be applied for big data (BD) communication over a Hybrid Ring-Mesh Protocol (HRMP) that mixes wired and wireless data exchange, to decrease the exchanged amount of transmitted data remarkably, increase the mesh coverage area, and decrease the number of connection wires, and decrease the network construction and maintenance cost. The proposed CoNC HRMP decreases the number of required wires from $M^{2}$ to $2 M$ as $M$ wires are needed between the $M$ members in the ring protocol (RP) plus $M$ wires between the $M$ members and the BS.

The results that have been obtained by MATLAB analysis confirm that applying CoNC over HRMP exploits the advantage of the RP and the PER improved significantly, for example, from 0.1 for two single stages to 0.04 for single and Odd-Even stages, at a channel erasure probability of 0.1 . The proposed algorithms created such a useful tradeoff between the data rate and PER, which indirectly means a trade-off between the data rate and the coverage region. Finally, the proposed algorithms managed to solve the problem when one or two wires got disconnected with PER better than the selfish mode, resulting in improving the network's reliability.

Based on above, the authors are proposing applying the proposed protocols over Multi-Protocol Label Switching for Traffic Engineering (MPLS-TE) technology [34-36]. Moreover, the queuing algorithms of FIFO, PQ, CQ, and WFQ in [37] are planned to be investigated to determine the performance after applying the proposed protocols. Finally, applying the proposed protocols over OFDM, DFrFT-OCDM, and MIMO-OFDM/ OCDM for the work published in [38-40] is regarded as another direction for future work. 


\section{Abbreviations}

BD: Big data; CoNC: Cooperation Network coding; WRT: Wired ring topology; WMN: Wireless mesh network; BS: Base station; PER: Packet error rate; HRMP: Hybrid Ring-Mesh Protocol; loT: Internet of things; loV: Internet of vehicles.

\section{Acknowledgements}

Not applicable.

\section{Authors' contribution}

HHA, AAAS, and MRK participated in mathematical design of the proposed method, and HHA and AAAS particularly worked on its computer implementation. HHA, CC, and MRK coordinated industrial application and data preparation and helped out for study. HHA and AA have completed the first draft of this paper. CC and MRK edited the draft for general write-up. All authors read and approved the final manuscript.

\section{Funding}

Not applicable.

Availability of data and materials

All the data and computer programs are available.

\section{Declaration}

\section{Competing interests}

The authors declare that they have no competing interests.

\section{Author details}

${ }^{1}$ Department of Energy Engineering, Zarqa University, Zarqa, Jordan. ${ }^{2}$ Department of Electrical and Electronics Engineering, Istanbul Gelisim University, Istanbul, Turkey. ${ }^{3}$ Department of Computer Science, College of Computer Science and Information Technology, Imam Abdulrahman Bin Faisal University, Dammam, Saudi Arabia. ${ }^{4}$ Department of Electronics and Communication Engineering, Birla Institute of Technology, Mesra, Ranchi, India. ${ }^{5}$ Department of Computer Engineering, Persian Gulf University, Bushehr, Iran.

Received: 30 March 2021 Accepted: 19 July 2021

Published online: 27 July 2021

\section{References}

1. K. Bakshi, Considerations for big data: architecture and approach, in Proceedings of 2012 IEEE Aerospace Conference, Big Sky, MT, USA, 2012, pp. 1-7 (2012)

2. X. Chen, M. Xie, A split-and-conquer approach for analysis of extraordinarily large data. Stat. Sin. 24(4), 1655-1684 (2014)

3. H.V. Jagadish, J. Gehrke, A. Labrinidis, Y. Papakonstantinou, J.M. Patel, R. Ramakrishnan, C. Shahabi, Big data and its technical challenges. Commun. ACM 57(7), 86-94 (2014)

4. M.S. Mahmud, J.Z. Huang, S. Salloum, T.Z. Emara, K. Sadatdiynov, A survey of data partitioning and sampling methods to support big data analysis. Big Data Min. Anal. 3(2), 85-101 (2020). https://doi.org/10.26599/BDMA.2019.90200 15

5. L. Jin, M. Xing, R. Wang, Operation framework of the command information system based on big data analysis, in 2020 IEEE 5th International Conference on Cloud Computing and Big Data Analytics (ICCCBDA), Chengdu, China, 2020, pp. 459-462. https://doi.org/10.1109/ICCCBDA49378.2020.9095568.

6. T.Z. Emara, J.Z. Huang, A distributed data management system to support large-scale data analysis. J. Syst. Softw. $148,105-115(2019)$

7. H.H. Attar, D. Vukobratovic, L. Stankovic, V. Stankovic, Performance analysis of node cooperation with network coding in wireless sensor networks, in 2011 4th IFIP International Conference on New Technologies, Mobility and Security, Paris, 2011, pp. 1-4. https://doi.org/10.1109/NTMS.2011.5721048

8. H. Attar, L. Stankovic, V. Stankovic, Cooperative network-coding system for wireless sensor networks. IET Commun. 6(3), 344-352 (2012)

9. M. El-M, H. Attar, A.A.A. Solyman, L. Stankovic, Network coding cooperation performance analysis in wireless network over a lossy channel, M users and a destination scenario. Commun. Netw. 8, 257-280 (2016). https://doi.org/ $10.4236 /$ cn.2016.84023

10. S. Nazir, V. Stankovic, H. Attar, L. Stankovic, S. Cheng, Relay-assisted rateless layered multiple description video delivery. IEEE J. Sel. Areas Commun. 31(8), 1629-1637 (2013)

11. H. Attar, L. Stankovic, M. Alhihi, and A. Ameen, Deterministic network coding over long term evaluation advance communication system, in Proceedings of 4th International Conference on Digital Information and Communication Technology and its Application (DICTAP), May 2014, pp. 56-61.

12. H.H. Attar, A.A. Solyman, M.R. Khosravi, L. Qi, M. Alhihi, P. Tavallali, Bit and packet error rate evaluations for half-cycle stage cooperation on 6G wireless networks. Phys. Commun. 44, 101249 (2021). https://doi.org/10.1016/j.phycom. 2020.101249

13. H. Attar, M. Alhihi, B. Zhao, L. Stankovic, Network coding hard and soft decision behavior over the physical payer using PUMTC, in 2018 International Conference on Advances in Computing and Communication Engineering (ICACCE), Paris, 2018, pp. 471-474. https://doi.org/10.1109/ICACCE.2018.8441742.

14. S. K. Jha, P. K. Jana, A new distributed approach for building balanced ring for fault tolerance in mesh architecture, in 2009 Proceeding of International Conference on Methods and Models in Computer Science (ICM2CS), Delhi, 2009, pp. 1-4. https://doi.org/10.1109//CM2CS.2009.5397940 
15. W. Zheng, X. Xie, Z. You, Y. Chen, X. Yu, Research on ring-based routing for wireless mesh network, in 2010 International Conference on Anti-Counterfeiting, Security and Identification, Chengdu, 2010, pp. 347-350. https://doi.org/10. 1109/ICASID.2010.5551352.

16. D. Jin, K. Wang, L. Feng, Capacity and coverage analysis of ring wireless mesh network, in Proceedings of 2012 International Conference on Measurement, Information and Control, Harbin, 2012, pp. 967-970. https://doi.org/10.1109/MIC. 2012.6274024

17. C. Yu, M. Ku, H. Lin, A hybrid mesh-ring topology for bluetooth networks, in 2018 IEEE 5 G World Forum (5GWF), Silicon Valley, CA, 2018, pp. 520-523.https://doi.org/10.1109/5GWF.2018.8517076

18. Y. Peng, Q. Deng, L. Guo, F. Wang, A new network coding based routing protocol for enhancing throughput capacity in wireless mesh networks. Chin. J. Electron. 28(2), 416-422 (2019). https://doi.org/10.1049/cje.2019.01.015

19. W. F. Sheng, Network coding method for self-similar streaming media flow in wireless mesh network, in 2018 International Conference on Engineering Simulation and Intelligent Control (ESAIC), Changsha, 2018, pp. 334-339. https:// doi.org/10.1109/ESAIC.2018.00084

20. A. Rezaei, L. Farzinvash, Online QoS multicast routing in multi-channel multi-radio wireless mesh networks using network coding, in 2019 9th International Conference on Computer and Knowledge Engineering (ICCKE), Mashhad, Iran, 2019, pp. 53-59. https://doi.org/10.1109/ICCKE48569.2019.8964980

21. J. M. Castillo-Secilla, P. C. Aranda, F. J. B. Outeiriño, J. Olivares, Experimental procedure for the characterization and optimization of the power consumption and reliability in zigbee mesh networks, in 2010 Third International Conference on Advances in Mesh Networks, Venice, 2010, pp. 13-16. https://doi.org/10.1109/MESH.2010.16

22. N. Tatebe, K. Hattori, T. Kagawa, Y. Owada, K. Hamaguchi, Energy-efficient construction algorithm for mobile mesh networks, in The 20th Asia-Pacific Conference on Communication (APCC2014), Pattaya, 2014, pp. 73-77. https://doi.org/ 10.1109/APCC.2014.7091608

23. R. Kashyap, M. Azman, J.G. Panicker, Ubiquitous mesh: a wireless mesh network for loT systems in smart homes and smart cities, in 2019 IEEE International Conference on Electrical, Computer and Communication Technologies (ICECCT), Coimbatore, India, 2019, pp. 1-5. https://doi.org/10.1109//CECCT.2019.8869482

24. J. Kaur, M.A. Khan, M. Iftikhar, M. Imran, Q. EmadUIHaq, Machine learning techniques for $5 \mathrm{G}$ and beyond. IEEE Access 9, 23472-23488 (2021). https://doi.org/10.1109/ACCESS.2021.3051557

25. M. Servetnyk, C.C. Fung, Z. Han, Unsupervised federated learning for unbalanced data, in GLOBECOM 2020-2020 IEEE Global Communications Conference, Taipei, Taiwan, 2020, pp. 1-6. https://doi.org/10.1109/GLOBECOM42002. 2020.9348203

26. H. Attar, Physical layer deterministic network coding using PUM turbo codes over AWGN channel, N nodes through a base station scenario. Commun. Netw. 8, 241-256 (2016)

27. S. Wan, R. Gu, T. Umer et al., Toward offloading internet of vehicles applications in $5 \mathrm{G}$ networks. IEEE Trans. Intell. Transp. Syst. (2020). https://doi.org/10.1109/TITS.2020.3017596

28. X. Xu, X. Liu, Z. Xu et al., Joint optimization of resource utilization and load balance with privacy preservation for edge services in $5 G$ networks. Mob. Netw. Appl. 25, 713-724 (2020)

29. X. Xu, Y. Xue, L. Qi et al., Load-aware edge server placement for mobile edge computing in $5 \mathrm{G}$ networks, in International Conference on Service-Oriented Computing, pp. 494-507 (2019)

30. S. Wan, X. Li, Y. Xue et al., Efficient computation offloading for internet of vehicles in edge computing-assisted $5 \mathrm{G}$ networks. J. Supercomput. 76, 2518-2547 (2020)

31. M. Abbasi, H. Rezaei, V.G. Menon et al., Enhancing the performance of flow classification in SDN-based intelligent vehicular networks. IEEE Trans. Intell. Transp. Syst. (2020). https://doi.org/10.1109/TITS.2020.3014044

32. M. Abbasi, A. Shokrollahi et al., High-performance flow classification using hybrid clusters in software defined mobile edge computing. Comput. Commun. 160, 643-660 (2020)

33. M. Abbasi, M.R. Khosravi, A robust and accurate particle filter-based pupil detection method for big datasets of eye video. J. Grid Comput. 18(2), 305-325 (2020)

34. M. Alhihi, M. Khosravi, H. Attar, M. Samour, Determining the optimum number of paths for realization of multi-path routing in MPLS-TE networks. Telkomnika 15(4), 1701-1709 (2017). https://doi.org/10.12928/TELKOMNIKA.v15i4 6597

35. H. Attar, Multipath routing mathematical model to solve the traffic engineering in multi-protocol label switching network. J. Comput. Commun. 5, 113-122 (2017)

36. H. Attar, M. Alhihi, M. Samour, A.A.A. Solyman, S.S. Igorovich, K.N. Georgievna, F. Khalil, A mathematical model for managing the distribution of information flows for MPLS-TE networks under critical conditions. Commun. Netw. 10 31-42 (2018)

37. H. Attar, M.R. Khosravi, S.S. Igorovich, K.N. Georgievan, M. Alhihi, Review and performance evaluation of FIFO, PQ, CQ FQ, and WFQ algorithms in multimedia wireless sensor networks. Int. J. Distrib. Sens. Netw. (2020). https://doi.org/10 $1177 / 1550147720913233$

38. H.H. Attar, A.A.A. Solyman, A.E.F. Mohamed, M.R. Khosravi, V.G. Menon, A.K. Bashir, P. Tavallali, Efficient equalisers for OFDM and DFrFT-OCDM multicarrier systems in mobile E-health video broadcasting with machine learning perspectives. Phys. Commun. 42, 101173 (2020)

39. A.A. Solyman, H. Attar, M.R. Khosravi, B. Koyuncu, MIMO-OFDM/OCDM low-complexity equalization under a doubly dispersive channel in wireless sensor networks. Int. J. Distrib. Sens. Netw. (2020). https://doi.org/10.1177/15501 47720912950

40. A.A.A. Solyman et al., A low-complexity equalizer for video broadcasting in cyber-physical social systems through handheld mobile devices. IEEE Access 8, 67591-67602 (2020). https://doi.org/10.1109/ACCESS.2020.2982001

\section{Publisher's Note}

Springer Nature remains neutral with regard to jurisdictional claims in published maps and institutional affiliations. 